\title{
LESIÓN DE LA MÉDULA ESPINAL. ACTUALIZACIÓN BIBLIOGRÁFICA: FISIOPATOLOGÍA Y TRATAMIENTO INICIAL
}

\author{
LESÃO DE MEDULA ESPINAL. ATUALIZAÇÃO DA LITERATURA: FISIOPATOLOGIA E TRATAMENTO INICIAL \\ SPINAL CORD INJURY. LITERATURE UPDATE: PHYSIOPATHOLOGY AND INITIAL TREATMENT
}

Vicente Ballesteros Plaza ${ }^{1}$, Bartolomé Marré Pacheco¹, Celmira Martínez Aguilar ${ }^{1}$, José Fleiderman Valenzuela1 ${ }^{1}$, Juan José Zamorano Pérez ${ }^{1}$

\begin{abstract}
RESUMEN
La fisiopatología del trauma raquimedular (TRM) es compleja y aún no se conoce completamente. La lesión al cordón espinal está determinada por procesos primarios y secundarios. La lesión primaria se debe a la transmisión de energía mecánica a la médula y las estructuras neurales durante el evento traumático. La lesión secundaria, que compromete estructuras que habían permanecido indemnes después del trauma inicial, desencadena alteraciones en: la perfusión microvascular, la liberación de radicales libres y de neurotransmisores, la peroxidación lipídica, la concentración iónica y la consecuente muerte celular tanto por necrosis como por apoptosis. La investigación en el tratamiento del TRM, basada en el conocimiento actual de estos mecanismos de lesión, ha buscado el desarrollo de intervenciones terapéuticas tempranas que atenúen el efecto de estos mecanismos fisiopatológicos secundarios, tanto en el sitio del accidente, como después del ingreso a un centro de trauma. Dentro de la intervención farmacológica se ha descrito, por su teórico efecto protector en el pronóstico neurológico de los pacientes con TRM, el uso de metil-prednisolona, gangliósidos y medicamentos antagonistas de los opiáceos, del receptor de glutamato y de los canales iónicos. Sin embargo, aún no se ha identificado ninguna intervención que modifique este pronóstico en forma significativa.
\end{abstract}

Descriptores: Lesiones/ historial de la médula espinal/historia; Lesiones/ etiología de la médula espinal; Lesiones/ patofisiología de la médula espinal; Revisión.

\section{RESUMO}

A fisiopatologia da lesão de medula espinal (LME) é complexa e não está completamente esclarecida. A LME é determinada por processos primários e secundários. A lesão inicial é produzida pela transmissão de energia mecânica para a medula espinal e as estruturas neurais. A lesão secundária atua sobre as estruturas que são poupadas pelo trauma inicial, afetando a perfusão microvascular e as concentrações iônicas, desencadeando a liberação de radicais livres e neurotransmissores e ativando a peroxidação lipídica, o que produz a morte celular. A pesquisa recente sobre o tratamento da LME concentra-se no desenvolvimento de intervenções oportunas que possam atenuar os efeitos dos mecanismos fisiopatológicos secundários. Entre as intervenções farmacológicas, têm sido usados: metilprednisolona, gangliosídeos e antagonistas de opiáceos, de receptores de glutamato e de canais iônicos, considerando seu efeito protetor teórico sobre o prognóstico neurológico dos pacientes com LME. Contudo, nenhuma dessas intervenções modificou significativamente esse prognóstico até agora.

Descritores: Traumatismos da medula espinal/história; Traumatismos da medula espinal/etiologia; Traumatismos da medula espinal/fisiopatologia; Revisão.

\begin{abstract}
The pathophysiology of spinal cord injury (SCI) is complex and still not fully elucidated. Primary and secondary processes determine SCl. The initial injury is produced by the transmission of the mechanical energy of the trauma to the spinal cord and neural structures. The secondary injury acts on those structures that were spared by the initial trauma, affecting microvascular perfusion and ionic concentrations, inducing the liberation of free radicals and neurotransmitters and activating lipid peroxidation, thus producing cellular death. Recent research in the treatment of SCl is focused on the development of timely therapeutic interventions that could attenuate the effects of these secondary pathophysiological mechanisms. Among the pharmacological interventions, methyl-prednisolone, gangliosides and antagonists of opiod, glutamate receptor and ionic channels have been used, advocating their theoretical protective effect in the neurological prognosis of patients with SCl. Nevertheless, none of these interventions has yet significantly modified this prognosis.
\end{abstract}

Keywords: Spinal cord injuries/history; Spinal cord injuries/etiology; Spinal cord injuries/physiopathology; Review.

\section{INTRODUCCIÓN}

Con el propósito de hacer más efectivo el tratamiento del trauma raquimedular, se ha buscado un mejor y detallado entendimiento de los procesos fisiopatológicos que ocurren después de este y de las interacciones entre sus componentes para producir las alteraciones funcionales que se observan. Este conocimiento ha mostrado que varios elementos de la respuesta natural al trauma, especialmente la inflamación, actúan acentuando el daño neurológico producido por el trauma original.
La mejor descripción de la fisiopatología del TRM presenta procesos primarios y secundarios durante su desarrollo. La lesión medular primaria (Figura 1) comprende el trauma mecánico inicial por la transmisión de la energía al cordón espinal, la deformidad secundaria y la compresión persistente debida al mismo. En general, se produce disrupción axonal, vascular y de las membranas celulares desencadenando la muerte celular y los cambios vasculares progresivos que inician la lesión medular secundaria, en la cual, la disfunción vascular, el edema y la isquemia, producen una serie

1. Equipo de Columna Vertebral. Servicio de Ortopedia y Traumatología. Hospital del Trabajador de Santiago.

Trabalho realizado no Hospital del Trabajador de Santiago Ramón Carnicer 185, Providencia, Santiago, Chile.

Correspondência: Hospital del Trabajador de Santiago Ramón Carnicer 185, Providencia, Santiago, Chile. 7501239. vicenteballesteros@gmail.com 


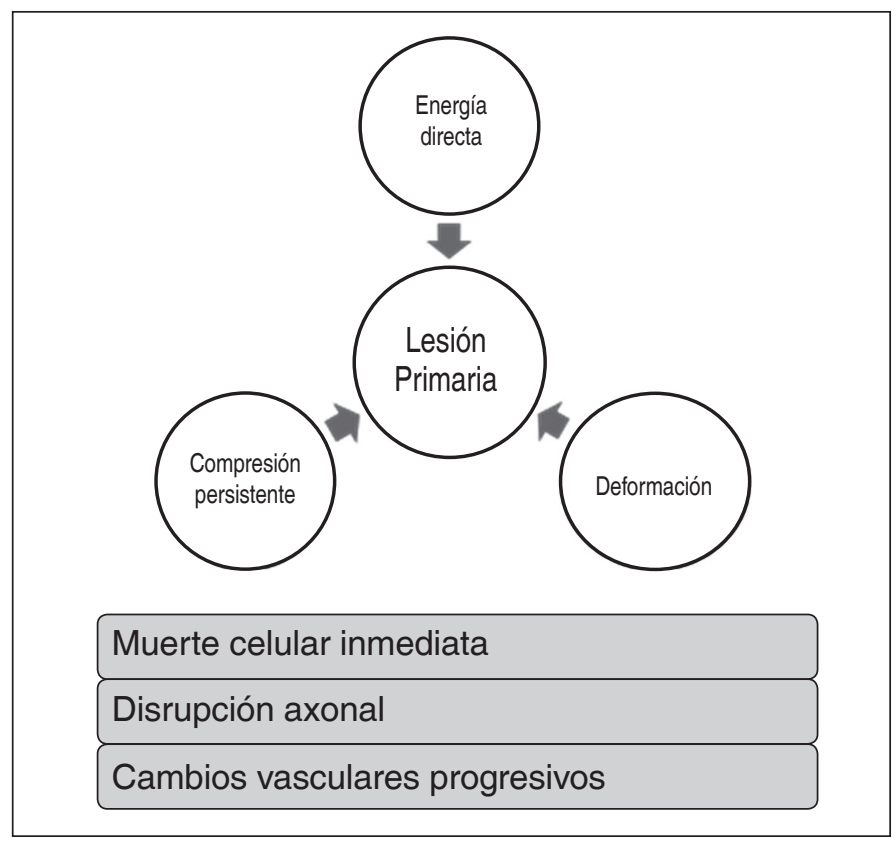

Figura 1. Mecanismos de la lesión primaria.

de procesos como la liberación de radicales libres, las alteraciones hidroelectrolíticas con excito-toxicidad y la apoptosis celular tardía, que resultan en un periodo sostenido de destrucción tisular.

Dado que es muy difícil realizar estudios patológicos en tejido humano postmortem, la mayoría del conocimiento obtenido hasta este momento en este sentido, se ha logrado en estudios animales lo que implica ambigüedad de las conclusiones a las que se ha llegado y limitaciones para la aplicación clínica'.

Esta revisión tiene como objeto describir el estado actual del conocimiento de la fisiopatología del TRM y de las intervenciones terapéuticas que se han descrito dentro de su tratamiento inicial.

\section{Mecanismos de la lesión medular primaria}

El mecanismo más común del TRM es el tipo compresivo-contusivo en el cual, el desplazamiento de los elementos de la columna vertebral, fragmentos óseos, discos y ligamentos, transmite energía mecánica sobre el cordón medular que frecuentemente se sostiene a través del tiempo. Otros mecanismos descritos son: cizallamiento, laceración, estiramiento agudo y aceleración-desaceleración súbitas.

Estos mecanismos físicos raramente producen un corte transversal de la médula completa con pérdida de la continuidad anatómica del cordón espinal².

\section{Mecanismos de la lesión medular secundaria}

El trauma desencadena una serie de procesos fisiopatológicos que producen la lesión medular secundaria (Figura 2). Para mejorar su comprensión y pensando en las implicaciones terapéuticas, ésta se ha subdividido en varias fases, de acuerdo con la cascada de eventos que se desencadenan en cada una de ellas ${ }^{3}$.

\section{Fase Inmediata (0 - 2 horas)}

Se inicia en el momento del trauma y está dominada por el compromiso relacionado directamente con la lesión inicial.

El primer cambio detectable es la inflamación generalizada del cordón espinal frecuentemente acompañada de hemorragia de la sustancia gris central en cuyas células se presenta necrosis debida a la disrupción mecánica de las membranas y/o a la isquemia resultante de la disrupción vascular ${ }^{4}$. La disrupción microvascular induce hemorragia en la sustancia blanca circundante que puede empeorar el compromiso local y extenderlo a segmentos adyacentes proximal y distalmente.

Llama la atención que a pesar de todas estas variaciones inflamatorias, se ha descrito que en este periodo no se observan anor-

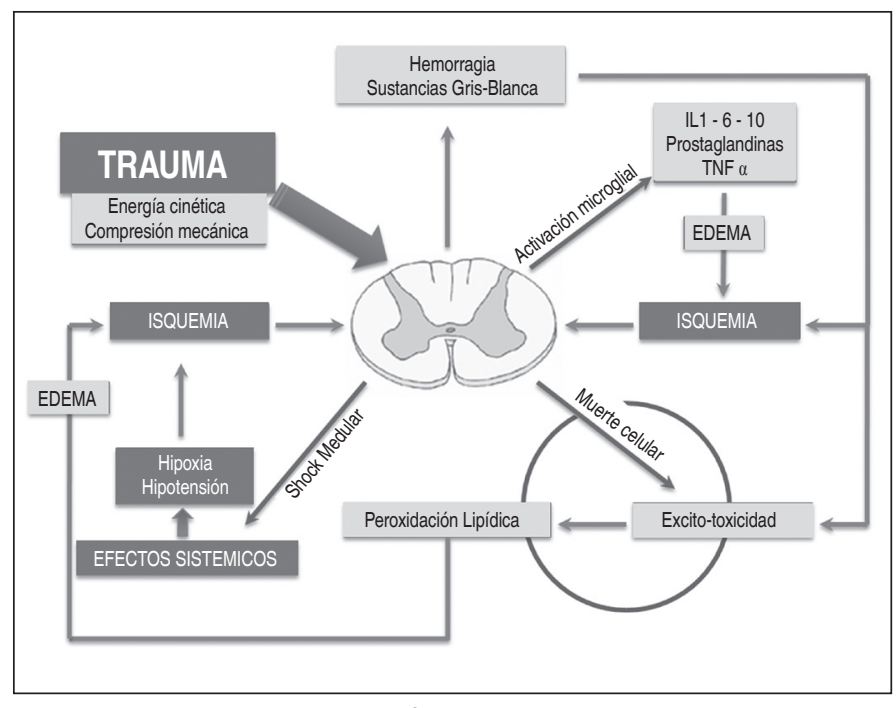

Figura 2. Mecanismos de la lesión medular secundaria.

malidades en la imagen por resonancia magnética. ${ }^{5}$ Sin embargo, en nuestra experiencia, con resonancia magnética de alta definición (3.0 Tesla), hemos observado imágenes medulares de alta intensidad en la secuencia T2, antes de las dos horas de evolución.

\section{Fase Aguda (2 - 48 horas)}

Es el periodo en el que la lesión secundaria se vuelve dominante. En esta fase es donde hay más susceptibilidad para realizar intervenciones neuro-protectoras. En las siguientes secciones se describirán los elementos de la cascada de la lesión medular secundaria.

\section{Alteración de la regulación iónica y excito-toxicidad}

La pérdida de la homeostasis iónica es una característica fundamental de la muerte celular por apoptosis y por necrosis; específicamente la alteración de la concentración de calcio ${ }^{6}$,que inicia una serie de procesos nocivos como la activación de calpainas, disfunción mitocondrial y producción de radicales libres.

La excito-toxicidad es el resultado de la activación excesiva de los receptores de glutamato porque su concentración se eleva súbitamente al liberarse en la lisis celular. Esto genera una falla del mecanismo de transporte intra y extracelular dependiente de energía, lo que perpetúa la alteración en la concentración iónica tisular?.

\section{Lesión mediada por radicales libres}

Altas concentraciones de radicales libres activan la peroxidación de ácidos grasos lo que produce lesión de las membranas celulares que implica muerte celular asociada a disfunción de los organelos y contribuye con la alteración de la homeostasis del calcio. Es decir, forman un círculo vicioso de procesos que aumentan su producción ${ }^{8-9}$.

\section{Permeabilidad de la Barrera Hemato-Medular (BH-M)}

La BH-M (equivalente a la Barrera Hemato-Encefálica en el segmento medular) es un filtro altamente selectivo que limita el transporte de compuestos desde y hacia el parénquima del sistema nervioso central (SNC). Después del TRM se produce un marcado incremento en la permeabilidad de la $\mathrm{BH}-\mathrm{M}$ debido al efecto endotelial del incremento local de mediadores inflamatorios ${ }^{10}$.

\section{Mediadores inflamatorios y respuesta inmune celular}

El proceso inflamatorio que sobreviene al TRM es altamente complejo y compromete numerosas poblaciones celulares que incluyen astrocitos, microglía, linfocitos T, neutrófilos y monocitos. De manera adicional, una multitud de mediadores intercelulares, como el factor de necrosis tumoral alfa, los interferones y las interleukinas, también juegan un papel importante. 
Dentro del reciente avance en la comprensión del complejo proceso neuro-inflamatorio, se han descubierto algunos aspectos deletéreos que empeoran el mecanismo de lesión secundaria y otros que contribuyen de manera beneficiosa en la recuperación de un ambiente propicio para el proceso regenerativo ${ }^{11}$. Este conflicto ha resaltado la importancia del reconocimiento adecuado de dichos aspectos con el fin de mejorar el tratamiento del TRM inhibiendo los procesos deletéreos y promoviendo los benéficos.

\section{Fase Subaguda (2 días a 2 semanas)}

En este periodo la respuesta fagocitaria es máxima buscando la remoción del tejido desbridado del área de lesión y la promoción, en algún grado, del crecimiento axonal ${ }^{12}$. También inicia una respuesta astrocitaria tardía en la cual, existe una hipertrofia e hiperplasia de la población de astrocitos localizados en la periferia de la lesión, formando un entrelazado proteico que va a configurar la cicatriz glial. Esta cicatriz representa una barrera tanto física como química para la regeneración axonal. Sin embargo, la reacción astrocitaria tardía promueve también el restablecimiento de la homeostasis iónica y de la integridad de la $\mathrm{BH}-\mathrm{M}^{13}$.

\section{Fase Intermedia (2 semanas a 6 meses)}

Se caracteriza por la continua maduración de la cicatriz glial y el crecimiento axonal regenerativo, aunque estos intentos son insuficientes para conseguir una recuperación funcional significativa, principalmente en lesiones severas ${ }^{14}$.

\section{Fase Crónica (6 meses en adelante)}

En esta se lleva a cabo la maduración y estabilización de la lesión. Continúa la formación de la cicatriz glial y se instaura de la degeneración Walleriana de los axones severamente lesionados (proceso que puede tardar años). Se considera que a los 2 años del TRM, la lesión esta completamente madura ${ }^{15}$

Lamentablemente la lesión no permanece estática y se puede producir una disfunción neurológica tardía secundaria a la formación de quistes y siringes hasta en el $30 \%$ de los pacientes. Esta disfunción se puede manifestar en compromiso ascendente, síntomas del tallo cerebral y dolor neuropático ${ }^{16}$.

Una consideración importante en el entendimiento de la evolución de los pacientes con un TRM, es que cada trauma es único tanto en causa y mecanismo como en la magnitud y el pronóstico del daño resultante. Aún existe desconocimiento de muchas de las vías de interacción de los procesos previamente descritos que dificultan un mejor desempeño terapéutico.

\section{Manejo inicial del TRM}

El manejo del TRM comienza en el mismo sitio donde sucede y debe cumplir estrictamente los protocolos del ATLS ${ }^{17-18}$. Existen tres objetivos fundamentales en la escena del trauma: prevenir lesiones adicionales, mantener oxigenación y perfusión adecuadas, a través de las maniobras de resucitación. También es crucial realizar un traslado oportuno y adecuado a un centro de referencia que esté provisto de un equipo multidisciplinario de profesionales y de la tecnología necesaria para responder a los requerimientos terapéuticos de este tipo de pacientes.

\section{Soporte Respiratorio}

Es fundamental mantener una adecuada oxigenación, ya que esta atenúa en forma importante el daño adicional debido a la isquemia resultante por la lesión inicial. Por otra parte, los pacientes con lesiones cervicales tienen compromiso inmediato en los volúmenes respiratorios debido al compromiso variable de la musculatura torácica.

Las complicaciones respiratorias están íntimamente relacionadas con la severidad del TRM y el compromiso sistémico. Un adecuado manejo de la vía aérea y de la terapia de higiene broncopulmonar previene fallas respiratorias posteriores y procesos infecciosos asociados ${ }^{19}$.

\section{Manejo Hemodinámico}

Es de vital importancia mantener una adecuada perfusión con el fin de optimizar el control de los mecanismos de la lesión me- dular secundaria. Los pacientes que sufren TRM se encuentran generalmente enmarcados dentro del politraumatismo; por lo tanto estos pacientes tienen dos potenciales causas de compromiso hemodinámico: la hipovolemia y el choque neurogénico que consiste en la pérdida del tono vasomotor como consecuencia del trauma expresado en forma primaria como una hipotensión generalizada.

\section{INTERVENCIÓN FARMACOLÓGICA PARA TRM}

\section{Esteroides}

Los mecanismos precisos por los cuales los esteroides tienen efecto neuroprotector, no están completamente esclarecidos. Sin embargo, se ha propuesto que inhiben la peroxidación de lípidos y las citoquinas inflamatorias, modulan la respuesta celular inmune-inflamatoria, mejoran la perfusión vascular y previenen la entrada y acumulación de calcio en las células. La metil-prednisolona (MP) parece ser la más eficaz comparada con otros glucocorticoides ${ }^{20}$.

El uso generalizado que actualmente tiene la MP esta basado en los Estudios del Trauma Espinal Agudo Nacional (NASCIS) I, II y III. En el primero se aleatorizaron 330 pacientes a recibir un esquema de dosis alta (1000 mg/día) o dosis baja (100 mg/día) de MP en las primeras 48 horas de evolución después del trauma. No se encontraron diferencias significativas en la recuperación neurológica al sexto mes de seguimiento ${ }^{21}$. En el segundo, se utilizó una dosis intermedia (bolo inicial de $30 \mathrm{mg} / \mathrm{K}$ seguido por una infusión continua de $5.4 \mathrm{mg} / \mathrm{K} / \mathrm{h}$ durante $23 \mathrm{hs}$ ) comparada con Naloxona y placebo en 487 pacientes en las primeras 12 horas de evolución después del trauma. Este estudio tampoco detectó diferencias en la recuperación neurológica a los seis meses de seguimiento entre los tres grupos ${ }^{22}$, sin embargo, en un análisis post hoc se observó que los pacientes a los que les iniciaron la terapia con MP en las primeras 8 horas después del trauma, presentaron una significativa recuperación neurológica motora y sensitiva a los seis meses y el año de seguimiento ${ }^{23}$. Finalmente, el tercer estudio, basado en los hallazgos del segundo, comparó la duración del tratamiento con MP entre 24 y 48 horas en 499 pacientes. Este trabajo no contó con grupo placebo por considerarlo anti-ético. La recuperación neurológica fue similar entre los grupos de estudio ${ }^{24}$. Nuevamente se realizó un análisis post hoc de los resultados encontrando que en los pacientes que se inició la terapia entre las tres y las ocho horas después del trauma y se mantuvo por 48 horas, hubo recuperación neurológica significativamente superior ${ }^{25}$

Dado que los resultados primarios de los estudios NASCIS fueron negativos y las diferencias encontradas se basaron en análisis post hoc, ha habido numerosos cuestionamientos al respecto de la interpretación de sus resultados e inclusive, en algunos centros de referencia, se ha descontinuado el uso de la MP. Adicionalmente se debe reconocer que el posible efecto benéfico de la MP estuvo atenuado por el aumento de la incidencia de complicaciones como infecciones de la herida quirúrgica, neumonía severa, sepsis y embolismo pulmonar, especialmente en el régimen de 48 horas del NASCIS III.

A pesar de estas críticas y de la publicación de una revisión sistemática en el 2000 en la que se afirma que la literatura disponible no soporta el uso de altas dosis de MP en el tratamiento del TRM agudo y que la evidencia no descarta su efecto deletéreo manifestado en posibles complicaciones ${ }^{26}$, la última actualización del grupo Cochrane al respecto del tema, sigue realizando las recomendaciones de tratamiento con MP en TRM basada en los resultados de los estudios NASCIS, específicamente con el esquema NASCIS $\|^{27}$.

\section{Gangliósidos}

Los gangliósidos son glicolípidos altamente expresados en las membranas celulares del SNC. Se ha descrito un efecto neuroprotector en su utilización en modelos experimentales animales basados en la teoría que aumentan el crecimiento y la plasticidad neuronal, inhiben la excito-toxicidad y previenen la apoptosis ${ }^{28}$.

El estudio piloto del GM1 o monosialotetrahexosilgangliósido $\left(\right.$ Sygen ${ }^{\circledR}$ ) realizado en 37 pacientes en 1991 tuvo resultados prometedores con respecto a la recuperación neurológica de los pacientes $^{29}$. Estos hallazgos impulsaron el desarrollo de un estudio multicéntrico aleatorizado entre 1992 y 1997. En esta investigación 
no se detectaron diferencias significativas entre los grupos de tratamiento (alta y baja dosis de GM1) y el grupo control con respecto a la recuperación motora o sensitiva, la frecuencia de eventos adversos o la mortalidad ${ }^{30}$

Desde el punto de vista clínico no se han realizado nuevos estudios del efecto del GM1 en el tratamiento del TRM por lo cual, y así como lo muestra la revisión de Cochrane con respecto al tema, no existe ninguna evidencia que soporte el uso habitual de los gangliósidos en el TRM ni que descarte un efecto perjudicial en los pacientes en los cuales podrían ser utilizados ${ }^{31}$.

\section{Antagonistas de los opiáceos}

La naloxona, antagonista inespecífico de los receptores de opiáceos, fue investigada extensamente en los años 80 después de estudios en animales que demostraron su efecto en disminuir la severidad del shock neurogénico y mejorar el flujo sanguíneo de la médula espinal sin lograr reproducirlo ${ }^{24}$.

La naloxona fue incluida en uno de los brazos de estudio del NASCIS II. En las conclusiones iniciales, se indicó que no concedió ningún beneficio terapéutico sobre el placebo, aunque posteriormente se justificaría este hallazgo con que la dosis utilizada fue subterapéutica ${ }^{26}$. Existe un reporte reciente de aparición de espasticidad severa asociada al uso de infusión de naloxona en pacientes con $\mathrm{TRM}^{32}$.

Antagonistas del receptor de Glutamato y de los canales iónicos

A finales de la década de los noventa, se estudió el potencial efecto neuroprotector de agente antiglutamatérgicos como el Selfotel $^{\circledR}$ (antagonista competitivo del receptor de N-metil- D-aspartato) sin éxito dada la significativa frecuencia de efectos adversos como agitación, sedación, alucinaciones y déficit de memoria ${ }^{33}$. Por lo tanto se empezó a explorar en un antagonista no competitivo del NMDA, la gaciclidina (GK11) que inicialmente tuvo resultados prometedores en estudios en animales. Sin embargo, el estudio fase Il (estudio piloto de evaluación de efectividad clínica) del producto falló en demostrar mejoría significativa en las escalas de la ASIA comparado con la terapia con placebo ${ }^{34}$

\section{REFERENCIAS}

1. Hagg T, Oduega M. Degenerative and spontaneous regenerative processes after spinal cord injury. J Neurotrauma. 2006;23(3/4):264-80

2. Sekhon LH, Fehlings MG. Epidemiology, demographics, and pathophysiology of acute spinal cord injury. Spine (Phila Pa 1976). 2001;26(24S):S2-12.

3. Rowland JW, Hawryluk GW, Kwon B, Fehlings MG. Current status of acute spinal cord injury physiophysiology and emerging therapies: promise on the horizon. Neurosurg Focus.2008;25 (5):E2-13

4. Tator $\mathrm{CH}$, Koyanagi I. Vascular mechanisms in the pathophysiology of human spinal cord injury. J Neurosurg. 1997:86(3):483-92.

5. Aoyama T, Hida K, Akino M, Yano S, Iwasaki Y, Saito H. Ultra-early MRI showing no abnormality in a fall victim presenting with tetraparesis. Spinal Cord. 2007;45(10):695-9.

6. Schanne FA, Kane AB, Young EE, Farber JL. Calcium dependence of toxic cell death: a final common pathway. Science. 1979;206(4419):700-2

7. Lipton SA, Rosenberg PA. Excitatory amino acids as a final common pathway for neurologic disorders. N Engl J Med. 1994;330(9):613-22.

8. Xiong Y, Rabchevsky AG, Hall ED. Role of peroxynitrite in secondary oxidative damage after spinal cord injury. J Neurochem. 2007;100(3):639-49.

9. Hall ED, Braughler JM. Free radicals in CNS injury. Res Publ Assoc Res Nerv Ment Dis. 1993;71:81-105

10. Schnell L, Fearn S, Schwab ME, Perry VH, Anthony DC. Cytokine-induced acute inflammation in the brain and spinal cord. J Neuropathol Exp Neurol. 1999:58(3):245-54.

11. Fleming JC, Norenberg MD, Ramsay DA, Dekaban GA, Marcillo AE, Saenz AD, et al. The cellular inflammatory response in human spinal cords after injury. Brain. 2006;129(Pt 12):3249-69.

12. Donnelly DJ, Popovich PG. Inflammation and its role in neuroprotection, axonal regeneration and functional recovery after spinal cord injury. Exp Neurol. 2008:209(2):378-88.

13. Herrmann JE, Imura T, Song B, Qi J, Ao Y, Nguyen TK, et al. STAT3 is a critical regulator of astrogliosis and scar formation after spinal cord injury. J Neurosci. 2008;28(28):7231-43.

14. Hill CE, Beattie MS, Bresnahan JC. Degeneration and sprouting of identified descending supraspinal axons after contusive spinal cord injury in the rat. Exp Neurol. 2001;171(1):153-69.

15. Ehlers MD. Deconstructing the axon: Wallerian degeneration and the ubiquitin-proteasome system. Trends Neurosci. 2004;27(1):3-6.

16. Stoodley MA. Pathophysiology of syringomyelia. J Neurosurg. 2000;92(6):1069-70.

17. Programa avanzado de apoyo vital en trauma para médicos - ATLS ${ }^{\circledR}$. Comité de Trauma del Colegio Americano de Cirujanos. 7th ed. Santiago: American College of Surgeons; 2009.

18. Schmidt OI, Gahr RH, Gosse A, Heyde CE. ATLS(R) and damage control in spine trauma. World J Emerg Surg. 2009;4:9.

19. Nockels RP. Nonoperative management of acute spinal cord injury. Spine (Phila Pa 1976). 2001;26(24 Suppl):S31-7.

20. Kwon BK, Tetzlaff W, Grauer JN, Beiner J, Vaccaro AR. Pathophysiology and pharmacologic treatment of acute spinal cord injury. Spine J. 2004;4(4):451-64.
Desde el punto de vista teórico, los bloqueadores de los canales de calcio tienen un efecto potencial en el tratamiento del TRM. De hecho en estudios experimentales se observa aumento del flujo sanguíneo espinal. En un estudio clínico francés en el cual los pacientes recibieron el esquema NASCIS II de MP solo, en combinación y comparado con nimodipino y con un grupo control que recibió placebo, no se demostraron diferencias significativas entre los cuatro grupos $^{35}$. Dado que existe un potencial riesgo de hipotensión con el detrimento que puede producir en las condiciones generales del paciente, no está recomendada su utilización.

Finalmente debemos mencionar al Tiralazad, un antioxidante evaluado en el NASCIS III que no demostró efecto superior al obtenido con el régimen de 24 horas de administración de MP aunque, sin ser significativo, se insinuó cierto efecto en la protección contra complicaciones sépticas.

Actualmente se siguen desarrollando programas y líneas de investigación que buscan una mejor compresión de la fisiopatología del TRM y potenciales intervenciones aún no descritas ampliamente.

\section{CONCLUSIONES}

La fisiopatología del TRM es altamente compleja. Aunque su conocimiento ha progresado recientemente, se desconocen todos los eventos desencadenados por el mismo y su cronología, y a través de ello identificar potenciales intervenciones para mejorar el pronóstico neurológico de los pacientes y el mejor momento para realizarlas.

El manejo inicial del TRM empieza en el mismo sitio del trauma y esta encaminado a optimizar las condiciones respiratorias y hemodinámicas del paciente.

Dentro de la intervención farmacológica temprana que busca mejorar el pronóstico neurológico de los pacientes con TRM aún no se ha identificado ninguna que lo cambie en forma significativa. El uso de la MP, ampliamente recomendada, es debatible y no completamente soportado por la evidencia actual.

21. Bracken MB, Collins WF, Freeman DF, Shepard MJ, Wagner FW, Silten RM, et al. Efficacy of methylprednisolone in acute spinal cord injury. JAMA. 1984;251(1):45-52.

22. Bracken MB, Shepard MJ, Collins WF, Holford TR, Young W, Baskin DS, et al. A randomized, controlled trial of methylprednisolone or naloxone in the treatment of acute spinal-cord injury. Results of the Second National Acute Spinal Cord Injury Study. N Engl J Med. 1990;322(20):1405-11.

23. Bracken MB, Shepard MJ, Collins WF Jr, Holford TR, Baskin DS, Eisenberg HM, et al. Methylprednisolone or naloxone treatment after acute spinal cord injury: 1-year follow-up data. Results of the second National Acute Spinal Cord Injury Study. J Neurosurg. 1992;76(1):23-31.

24. Bracken MB, Shepard MJ, HolfordTR, Leo-Summers L, Aldrich EF, Fazl M, et al. Administration of methylprednisolone for 24 or 48 hours or tirilazad mesylate for 48 hours in the treatment of acute spinal cord injury. Results of the Third National Acute Spinal Cord Injury Randomized Controlled Trial. National Acute Spinal Cord Injury Study. JAMA. 1997-277(20):1597-604.

25. Bracken MB, Shepard MJ, Holford TR, Leo-Summers L, Aldrich EF, Fazl M, et al. Methylprednisolone or tirilazad mesylate administration after acute spinal cord injury: 1-year follow up. Results of the third National Acute Spinal Cord Injury randomized controlled trial. J Neurosurg. 1998:89(5):699-706.

26. Short DJ, El Masry WS, Jones PW. High dose methylprednisolone in the management of acute spinal cord injury - a systematic review from a clinical perspective. Spinal Cord. 2000;38(5):273-86.

27. Bracken MB. Steroids for acute spinal cord injury. Cochrane Database Syst Rev. 2012;1:CD001046.

28. Skaper SD, Leon A. Monosialogangliosides, neuroprotection, and neuronal repair processes. J Neurotrauma. 1992;9(Suppl 2):S507-16.

29. Geisler FH, Dorsey FC, Coleman WP. Recovery of motor function after spinal-cord injury-a randomized, placebo-controlled trial with GM-1 ganglioside. N Engl J Med. 1991;324(26):1829-38

30. Geisler FH, Coleman WP, Grieco G, Poonian D; Sygen Study Group. The Sygen multicenter acute spinal cord injury study. Spine (Phila Pa 1976). 2001:26(24 Suppl):S87-98.

31. Chinnock P, Roberts I. Gangliosides for acute spinal cord injury. Cochrane Database Syst Rev. 2005;(2):CD004444.

32. Brackett NL, Ibrahim E, Krassioukov A, Lynne CM. Systemic naloxone infusion may trigger spasticity in patients with spinal cord injury: case series. J Spinal Cord Med. 2007;30(3):272-5.

33. Hawryluk GW, Rowland J, Kwon B. Fehlings MG. Protection and repair of the injured spinal cord: a review of completed, ongoing, and planned clinical trials for acute spinal cord injury. Neurosurg Focus. 2008;25(5):E14-29.

34. Tadie M, D'Arbigny P, Mathé J, Loubert G, Saint-Marc C, Menthonnex P, et al. Acute spinal cord injury: early care and treatment in a muticenter study with gacyclidine [abstract]. Soc Neurosci.1999;25:1090.

35. Pointillart V, Petitjean ME, Wiart L, Vital JM, Lassié P, Thicoipé M, et al. Pharmacological therapy of spinal cord injury during the acute phase. Spinal Cord. 2000;38(2):71-6. 